Trauma Berufskrankh 2010 • 12[Suppl 3]:303-307 DOI 10.1007/s10039-010-1656-1

Online publiziert: 8. Juli 2010

(c) Springer-Verlag 2010

\author{
A. Rahmanian-Schwarz $\cdot$ H.-E. Schaller \\ Klinik für Plastische, Hand-, Rekonstruktive und Verbrennungschirurgie, \\ Eberhard-Karls-Universität Tübingen
}

\title{
Ligamentäre Verletzungen der Handwurzel und deren Folgen
}

Seit der Erstbeschreibung der Instabilitäten an der Handwurzel durch Linscheid et al. [21] haben detaillierte anatomische Studien und Fortschritte in der bildgebenden Diagnostik zu einer enormen Verbesserung des Verständnisses und der Definition dieser Erkrankung geführt.

Aufgrund der komplexen Pathophysiologie der Handwurzelverletzungen ist für eine präzise und frühzeitige Diagnose die handchirurgische Erfahrung maßgeblich. Im klinischen Alltag werden bei der routinemäßigen Röntgenkontrolle des posttraumatischen Handgelenks überwiegend der Radius und das Kahnbein inspiziert und nach einer schnellen, oft undifferenzierten klinischen Untersuchung die Diagnose gestellt. Dadurch werden häufig minimale Veränderungen und versteckte Bandläsionen übersehen, die erst Monate oder Jahre später in einer Arthrose des Handgelenks enden. Die sozialökonomischen Folgen dieser Verletzungen sind nicht zu unterschätzen, v. a. wenn man bedenkt, dass ein junger, berufstätiger Patient mit seinem stabilen Knochenbau posttraumatisch weniger unter Frakturen, sondern vielmehr unter schwer diagnostizierbaren Bandläsionen leidet.

Nur durch eine gezielte Analyse des Traumas und des Pathomechanismus sowie eine Kombination differenzierter klinischer Untersuchungen und darauf basierender diagnostischer Hilfsmittel kann das exakte Ausmaß der Verletzung festgestellt werden. Genaue Kenntnisse der Anatomie und Kinematik des Handgelenks sowie gebräuchliche Klassifikationsmuster der karpalen Instabilität und frü- he Behandlungsoptionen können langwierige Folgeschäden verhindern.

\section{Anatomie}

Der normal funktionierende Karpus erlaubt der Hand, sich in Relation zum Unterarm präzise zu positionieren, und gewährleistet eine bemerkenswert stabile Kraftübertragung.

Als zwischengeschaltetes Segment (,intercalated segment“) zwischen Radius und distaler Handwurzelknochenreihe besteht die proximale Handwurzelreihe aus Skaphoid, Lunatum und Triquetrum. Dieses Segment vermittelt sowohl die Bewegung als auch die Kraftübertragung zwischen Hand und Unterarm (- Abb. 1). Die distale Handwurzelreihe als Verbindung zur Mittelhand wird aus Trapezium, Trapezoideum, Capitatum und Hamatum gebildet. Weder Muskeln noch Sehnen inserieren am Knochen der proximalen Handwurzelreihe [21]. Sie dient als mechanische Verbindung, die dem Handgelenk Rotationsstabilität verleiht, während die Funktion der distalen Handwurzelreihe v. a. in der Stabilisierung und Kraftverteilung liegt [9].

Aufgrund vieler Verbindungen zwischen den einzelnen Karpalknochen wird im Handgelenk eine gute Stabilität erreicht. Diese wird durch verschiedene Bänder verstärkt, die die Handwurzelknochen untereinander mit den Metakarpalia, dem Radius sowie der Ulna verbinden $[3,15]$.

Die Beweglichkeit der einzelnen Handwurzelknochen wird zum einen durch die Stellung der benachbarten Gelenkflächen, zum anderen aber auch durch die Bandstrukturen eingeschränkt. Beide Handwurzelreihen unterscheiden sich in ihrer Beweglichkeit. Die interossären Ligamente der distalen Reihe sind kräftig und bilden eine straffe Einheit, weshalb diese bei Bewegungen der Hand keine wesentliche Veränderung erfährt. Die Knochen der proximalen Reihe bilden eine halb-

\begin{tabular}{|c|c|c|c|}
\hline \multirow[t]{2}{*}{ Typ } & \multicolumn{2}{|l|}{ Name } & \multirow[t]{2}{*}{ Charakteristika } \\
\hline & Abkürzung & Bedeutung & \\
\hline I & CID & $\begin{array}{l}\text { "carpal instability } \\
\text { dissociative" }\end{array}$ & $\begin{array}{l}\text { Instabilität zwischen den Karpalia in derselben Karpal- } \\
\text { reihe }\end{array}$ \\
\hline II & CIND & $\begin{array}{l}\text { "carpal instability } \\
\text { nondissociative" }\end{array}$ & $\begin{array}{l}\text { Instabilität zwischen den beiden karpalen Reihen oder } \\
\text { zwischen einer karpalen Reihe und dem benachbarten } \\
\text { nächsten knöchernen System } \\
\text { Schaden des extrinsischen ligamentären Systems }\end{array}$ \\
\hline III & $\mathrm{CIC}$ & $\begin{array}{l}\text { "carpal instability } \\
\text { complex" }\end{array}$ & \\
\hline IV & „adaptive ca & & $\begin{array}{l}\text { SLAC (,scapholunate advanced collapse") und } \\
\left.\text { SNAC („,scaphoid nonunion advanced collapse }{ }^{\prime \prime}\right)\end{array}$ \\
\hline
\end{tabular}




\section{Handgelenk}

a

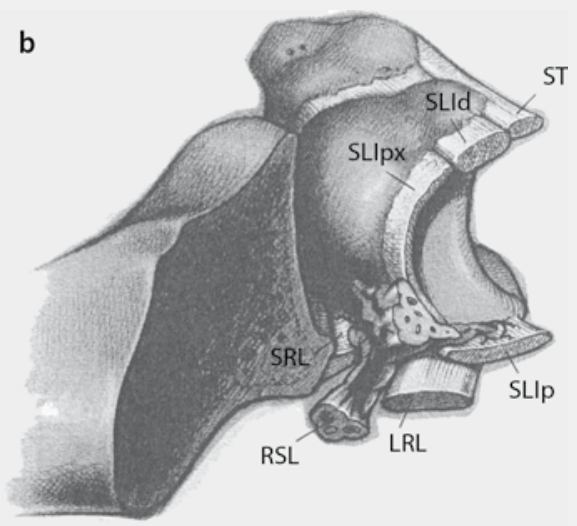

Abb. 14 Proximale Handwurzelreihe, $d$ dorsal, $L$ Lunatum, $L R L$ "long radiolunate ligament", $p$ palmar, $p x$ proximal, $R S L$,radioscapholunate ligament", S Skaphoid, SLI "scapholunate interosseus ligament", SRL "short radiolunate ligament", $S T$,"scaphotriquetral ligament", $T$ Triquetrum. (Nach [3])
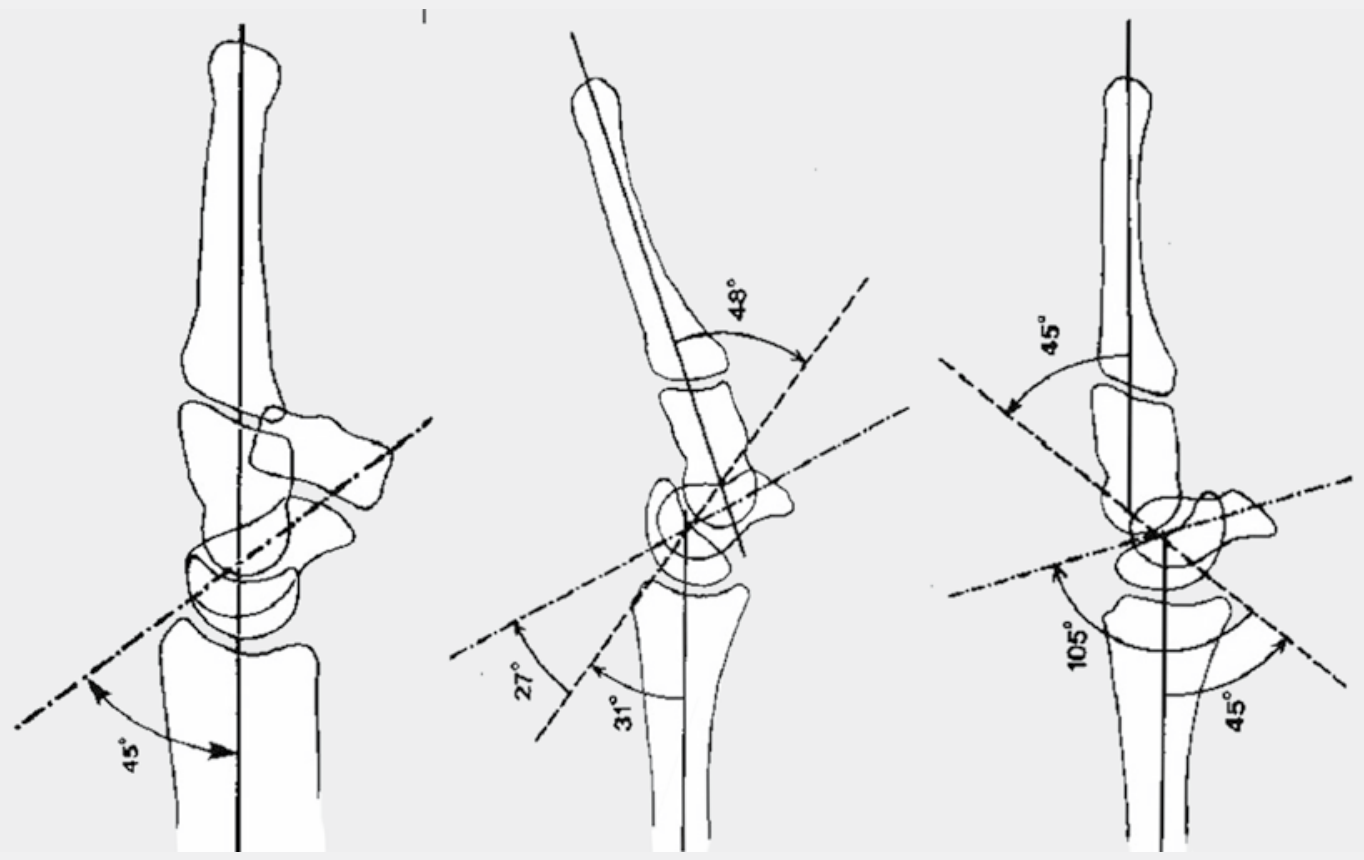

Abb. $2 \varangle$ Schematische Darstellung des normalen Handgelenks (links), PISIKonfiguration („,palmar intercalated segmental instability", Mitte) und DISI-Konfiguration ("dorsal intercalated segment instability", rechts)
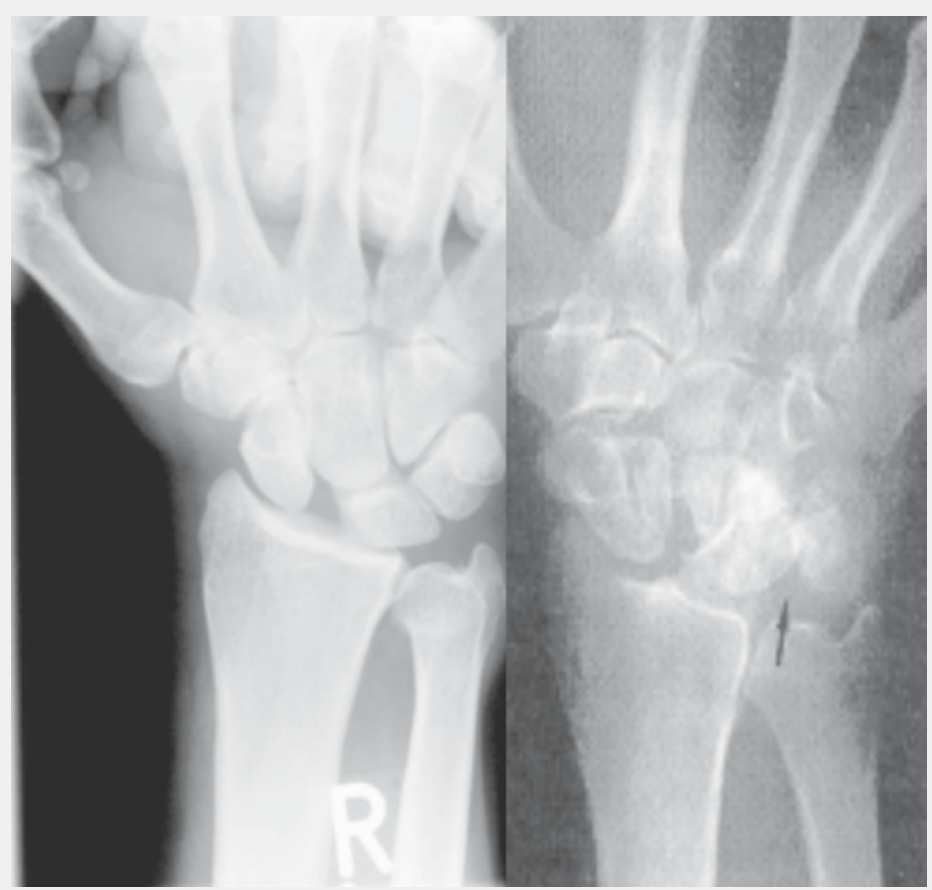

Abb. $3 \varangle$ Skapholunare Dissoziation links und lunotriquetrale Dissoziation rechts 
starre Einheit und haben daher bedeutend höhere Freiheitsgrade [1, 27].

\section{Klassifikation}

In Abhängigkeit von der einwirkenden Kraft, ihrer Lokalisation und der Position des Handgelenks kann ein Sturz auf das ausgestreckte Handgelenk eine Reihe unterschiedlicher Verletzungen bewirken. Bei Beteiligung der ligamentären Strukturen können Bandperforationen oder Risse mit noch intakter Bandfunktion bis hin zur kompletten Bandruptur und Dissoziation der benachbarten Knochen auftreten. In Abhängigkeit der Verletzungen der Bänder wird eine generelle Einteilung der karpalen Instabilität in 4 Typen beschrieben (• Tab. 1; $[2,12])$.

Eine Stauchung des Handgelenks in Extension, ulnarer Deviation und Supination kann zu einer perilunaren Instabilität führen. Dabei handelt es sich um ein progressives Verletzungsmuster, das vom skapholunaren über das kapitolunare bis zum lunotriquetralen Gelenk fortschreitet. Als Folge der lunotriquetralen Bandruptur kann das Triquetrum das Lunatum nicht mehr halten, es fällt unter dem Druck des Capitatums und aufgrund seiner Verbindung mit dem Skaphoid in eine flektierte Position.

Eine Ruptur des Lig. lunotriquetrum hat gewöhnlich eine Deformität des palmaren interkalierten Segments zur Folge, abgekürzt PISI („palmar intercalated segmental instability“). Bei dieser Konfiguration befindet sich das Lunatum in einer Flexion nach palmar mit einem Abfall des radiolunaren Winkels $<15^{\circ}$.

Bei einer Verletzung des Lig. scapholunatum kommt es ebenfalls durch die Torsionsstabilität im interkalierten Segment zur Achsenfehlstellungen des Lunatums. Es resultiert eine DISI-Konfiguration („dorsal intercalated segment instability") mit einem Anstieg der radiolunaren Winkel $>15^{\circ}$ (• Abb. 2; [4, 29]). Um die klinische Diagnose karpale Instabilität verifizieren zu können, muss in der seitlichen Röntgenaufnahme die Stellung der einzelnen Knochen durch Bestimmung ihrer Achsen und den sich daraus ergebenden Winkeln genau definiert werden

Die unterstützenden extrinsischen Bänder spielen eine wichtige Rolle bei

Trauma Berufskrankh 2010 - 12[Suppl 3]:303-307 DOI 10.1007/s10039-010-1656-1

c) Springer-Verlag 2010

\section{A. Rahmanian-Schwarz $\cdot$ H.-E. Schaller Ligamentäre Verletzungen der Handwurzel und deren Folgen}

\section{Zusammenfassung}

Bei der im klinischen Alltag üblichen routinemäßigen Röntgenkontrolle des posttraumatischen Handgelenks mit Inspektion von Radius und Kahnbein und einer schnellen, oft undifferenzierten klinischen Untersuchung werden minimale Veränderungen und versteckte Bandläsionen häufig übersehen mit erheblichen gesundheitlichen und sozialökonomischen Folgen. Nur durch eine gezielte Analyse des Traumas und des Pathomechanismus sowie eine Kombination differenzierter klinischer Untersuchungen und darauf basierender diagnostischer Hilfsmittel kann das exakte Ausmaß der Verletzung festgestellt werden. Für eine korrekte Diagnose- stellung sind genaue Kenntnisse der Anatomie und Kinematik des Handgelenks unverzichtbar. In Abhängigkeit der Verletzungen der Bänder werden 4 Typen der karpalen Instabilität unterschieden: CID ( ty dissociative"), CIND ("carpal instability nondissociative"), CIC ("carpal instability complex") und ",adaptive carpus". Je nach Instabilitätstyp kommen unterschiedliche Therapieverfahren in Betracht.

\section{Schlüsselwörter}

Radius · Karpus · Skapholunare Instabilität . Lunotriquetrale Instabilität .

Mediokarpale Instabilität

\section{Ligamentous wrist injury and its consequences}

\section{Abstract}

In routine clinical X-ray follow-up of posttraumatic wrist injury, including inspection of the radius and navicular bone and brief, often undifferentiated clinical examination, minimal changes and hidden ligament lesions can often be overlooked - with significant health-related and socioeconomic consequences. Only by targeted analysis of the trauma and its pathomechanism, as well as a combination of differentiated clinical examinations and diagnostic methods can the precise extent of injury be established. Exact knowledge of the anatomy and kinematics of the wrist is mandatory for correct diag- nosis. Depending on the ligament injury, distinction is made between four types of carpal instability: CID (carpal instability dissociative), CIND (carpal instability nondissociative), CIC (carpal instability complex) and adaptive carpus. Then, depending on the type of instability, a number of treatment methods may be considered.

\section{Keywords}

Radius · Carpus · Scapholunate instability . Lunotriquetral instability - Mediocarpal instability 


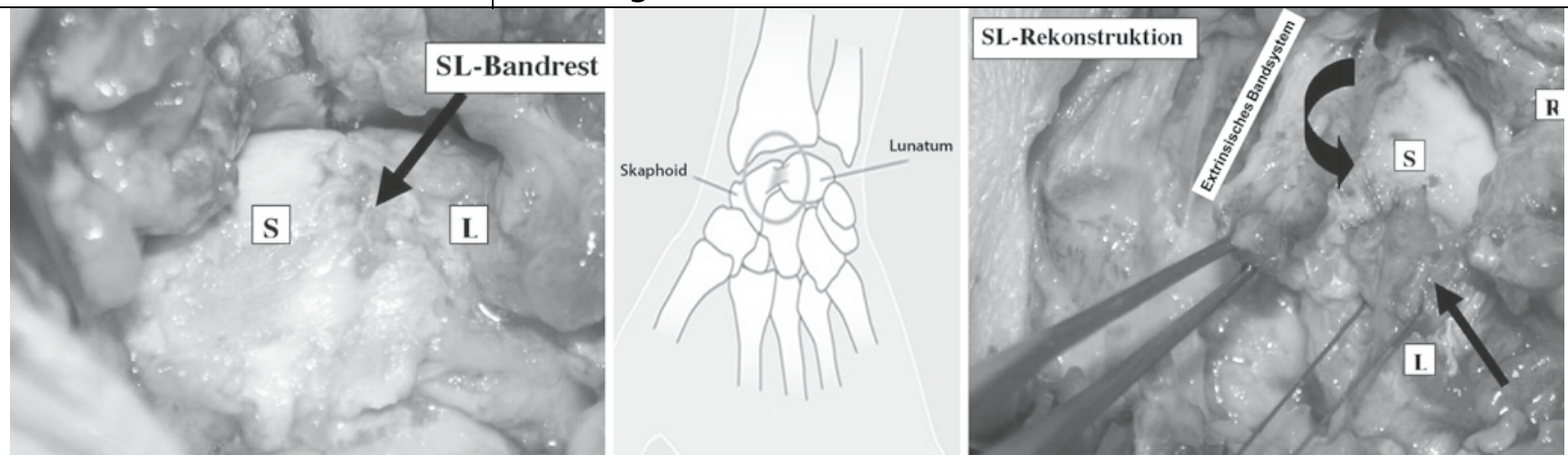

Abb. 4 \ Skapholunares Band, links intraoperativer Situs, rechts Bandrekonstruktion. S Skaphoid, L Lunatum, R Radius

der Prävention einer karpalen Instabilität, wenn bereits intrinsische Bandverletzungen vorliegen. Wie mehrere Kadaverstudien zeigten, löst eine isolierte Durchtrennung des skapholunaren Bandes noch keine radiologisch sichtbare Dissoziation aus $[4,22]$.

\section{Chirurgische Therapie}

Eine vollständige und detaillierte Darstellung aller Therapieoptionen der karpalen Instabilität kann im vorliegenden Beitrag nicht erfolgen. Um einen Überblick über die Behandlung dieser komplexen Verletzung zu verschaffen, wird jede Therapieform unter dem spezifischen Instabilitätstyp zusammengefasst.

\section{Skapholunare Instabilität}

Es gibt keinen Konsens über das beste Therapieverfahren, dieses sollte spezifisch nach dem vorliegenden Stadium oder Verletzungsgrad gewählt werden.

\section{Partielle Einrisse des skapholunaren Bandes}

Sie werden als okkulte oder prädynamische Instabilität interpretiert und können in der Regel konservativ behandelt werden.

\section{Komplette Banddurchtrennung}

Insbesondere bei einer Insuffizienz des extrinsischen Bandapparats kommt es zu einer akuten skapholunaren Dissoziation, die mit einer Instabilität und Spätfolgen einhergeht ( $\bullet$ Abb. 3).

Direkte Bandrekonstruktion. Optionen für die Therapie beinhalten die direkte Rekonstruktion mit oder ohne dorsale Kap- sulodese und temporären Arthrodesen (• Abb. 4). Diese Form der Behandlung eignet sich besonders für akute, nicht länger als 6 Wochen zurückliegende Einrisse, wenn ein ausreichender Rest des skapholunaren Bands vorhanden ist $[6,26]$.

Indirekte Bandrekonstruktion. Sie ist indiziert, wenn bei älteren Rupturen nicht mehr genügend Bandreste vorhanden sind und keine degenerative Arthrose zu beobachten ist. Sie beruht auf einer Stabilisierung des Skaphoids, um die rotatorische Subluxation zu korrigieren, die bei der skapholunaren Instabilität häufig vorkommt.

Die am weitesten verbreitete indirekte Bandrekonstruktion ist die dorsale Kapsulodese nach Blatt [5]. Bei dieser Technik verwendet man einen Bandstreifen der dorsalen Gelenkkapsel. Er wird am distalen Radius angebracht, wodurch sich die Handgelenkflexion um durchschnittlich $20 \%$ reduziert.

Die unterschiedlichen chirurgischen Techniken für die indirekte Rekonstruktion des skapholunaren Bands basieren entweder auf einem Sehnentransplantat (Bandplastik nach Brunelli u. Brunelli [7]) oder einem Knochen-Band-Knochen-Transplantat (Osteoligamentoplastik nach Weiss [31]) [25]. Jedes dieser Verfahren zeigt bis zu einem gewissen Grad Erfolg, doch ist dieser häufig nicht von Dauer. Sie erfordern eine lange dauernde Handgelenkimmobilisation und können zu Kontrakturen und Verlust der Beweglichkeit führen $[14,23]$.

\section{Arthrotische Veränderungen}

Liegen sie bereits vor, z. B. als Folge eines karpalen Kollapses, können die Karpektomie der proximalen Reihe bei noch in- takter Fovea lunata oder die Resektion des Skaphoids mit einer 4-Ecken-Arthrodese bei einer Radiokarpalarthrose zur Schmerzlinderung führen. Signifikante degenerative Veränderungen am proximalen Capitatum oder der Fovea lunata stellen eine Kontraindikation für eine Karpektomie der proximalen Reihe dar [13]. Hier besteht die chirurgische Therapieoption häufig in einer totalen Handgelenkfusion mit gleichzeitiger Denervation des Handgelenks nach Wilhelm [32].

\section{Lunotriquetrale Instabilität}

Für ihre Behandlung existiert kein einheitliches Regime. Der Behandlungsalgorithmus sollte sich nach Möglichkeit am Typ und am Alter der Verletzung orientieren. Während einige Autoren eine Immobilisationsperiode für akute Verletzungen empfehlen, befürworten andere eine arthroskopische Evaluation und eine direkte Bandrekonstruktion [20,30]. Bei Vorliegen einer lunotriquetalen Dissoziation empfiehlt sich eine direkte Bandnaht mit einer Kapsulodese (• Abb. 3).

Stellen sich Patienten erst lange Zeit nach ihrer initialen Verletzung vor, umfassen die chirurgischen Therapiemaßnahmen verschiedene Techniken wie

- sekundäre Bandrekonstruktion,

- Arthrodese,

- Kapsulodese oder

- ulnare Verkürzung.

Beschrieben ist eine Ligamentrekonstruktion durch einen distalen Streifen der Sehne des M. extensor carpi radialis [18, 19]. Da Patienten mit symptomatischer lunotriquetraler Instabilität oft auch ulnare Impaktionssyndrome aufweisen, behandelte Ruby [25] bei chronischer luno- 
triquetraler Ruptur ausschließlich mit einer ulnaren Verkürzung, besonders bei Vorliegen einer positiven oder neutralen Ulnavarianz.

Als eine weitere Behandlungsmethode wird die lunotriquetrale Fusion kontrovers diskutiert [8]. Pin et al. [24] und Kirschenbaum et al. [16] berichteten über etwas befriedigendere Ergebnisse nach einer solchen. Von 14 Patienten hatte nur einer andauernde Schmerzen; die durchschnittliche Griffstärke betrug 94\% im Vergleich zur kontralateralen Seite. Bei 2 Patienten ließ sich keine Fusion erzielen. Bei einem von ihnen wurde erneut eine Fusion durchgeführt, der andere war asymptomatisch. Die Bewegung im Handgelenk blieb in diesen Studien gut erhalten und betrug etwa $80-85 \%$ im Vergleich zum gesunden Handgelenk [16, 24]. Trotz der Resultate dieser beiden Studien berichteten andere Autoren über hohe Nichtheilungsraten, beispielsweise $57 \%$, sowie fortdauernden Schmerz bei $52 \%$ und eine verminderte Handgelenkbeweglichkeit bei 31\% ihrer Patienten [11, 28].

\section{Mediokarpale Instabilität}

Ihre Behandlung wird kontrovers diskutiert. Bei konservativ frustraner Therapie bewährte sich die Durchführung einer dorsalen Bandplastik im Sinne einer Verstärkung des Lig. radiocapitatum. Johnson u. Carrera [14] empfahlen die Fixierung des mittleren Anteils des Lig. radiocapitatum an das Lig. radiotriquetrum. Dies führt jedoch, wie häufig bei den Kapsulodesen, zur einer verminderten Extension des Handgelenks [10, 17].

\section{Weitere Verletzungen}

Bei in Fehlstellung verheilter distaler Radiusfraktur, bei Skaphoidpseudoarthrose oder Lunatumnekrose kann es zu einer statischen Fehlstellung zwischen der proximalen und der distalen Handwurzelreihe mit entsprechenden Sekundärveränderungen kommen. Als Rettungsoperation kann in diesen Fällen mit einer Korrekturosteotomie des Radius die karpale Instabilität behoben werden.

\section{Korrespondenzadresse}

\section{Dr. A. Rahmanian-Schwarz}

Klinik für Plastische, Hand-,

Rekonstruktive und Verbrennungschirurgie,

Eberhard-Karls-Universität Tübingen,

Schnarrenbergstraße 95, 72076 Tübingen

arahmanian@bgu-tuebingen.de

Interessenkonflikt. Der korrespondierende Autor gibt an, dass kein Interessenkonflikt besteht.

\section{Literatur}

1. Akahane M, Ono H, Nakamura T et al (2002) Static scapholunate dissociation diagnosed by scapholunate gap view in wrists with or without distal radius fractures. Hand Surg 7:191-195

2. Amadio M, Lucarelli L, Bellone M (1991) Cancer of the appendix. A report of 2 cases. Minerva Chir 46:1067-1070

3. Berger RA (1996) The gross and histologic anatomy of the scapholunate interosseous ligament. J Hand Surg [Am] 21:170-178

4. Berger RA, Landsmeer JM (1990) The palmar radiocarpal ligaments: a study of adult and fetal human wrist joints. J Hand Surg [Am] 15:847-854

5. Blatt G (1987) Capsulodesis in reconstructive hand surgery. Dorsal capsulodesis for the unstable scaphoid and volar capsulodesis following excision of the distal ulna. Hand Clin 3(1):81-102

6. Bloom HT, Freeland AE, Bowen V et al (2003) The treatment of chronic scapholunate dissociation: an evidence based assessment of the literature. Orthopedics 26:195-204

7. Brunelli GA, Brunelli GR (1995) A new technique to correct carpal instability with scaphoid rotary subluxation: a preliminary report. J Hand Surg [Am] 20(3 Pt 2):S82-S85

8. Craigen MA, Stanley JK (1995) Wrist kinematics. Row, column or both? J Hand Surg [Br] 20:165-170

9. Dobyns JHLR, Chao EYS (1975) Traumatic instability of the wrist. American Academy of Orthopaedic Surgeons. Instr Course Lect 182-199

10. Easterling KJ, Wolfe SW (1994) Scaphoid shift in the uninjured wrist. J Hand Surg [Am] 19:604-606

11. Garcia-Elias M, Ribe M, Rodriguez J et al (1995) Influence of joint laxity on scaphoid kinematics. J Hand Surg [Br] 20:379-382

12. Geissler WB, Freeland AE (1996) Arthroscopically assisted reduction of intraarticular distal radial fractures. Clin Orthop 327:125-134

13. Gilford WW, Bolton RH, Lambrinudi C (1943) The mechanism of the wrist joint with special reference to fractures of the scaphoid. Guys Hosp Rep 92:52-59

14. Johnson RP, Carrera GF (1986) Chronic capitolunate instability. J Bone Joint Surg Am 68:11641176

15. Jones WA (1988) Beware the sprained wrist. The incidence and diagnosis of scapholunate instability. J Bone Joint Surg Br 70:293-297

16. Kirschenbaum D, Coyle MP, Leddy JP (1993) Chronic lunotriquetral instability: diagnosis and treatment. J Hand Surg [Am] 18(6):1107-1112

17. Kuo CE, Wolfe SW (2008) Scapholunate instability: current concepts in diagnosis and management. J Hand Surg [Am] 33:998-1013

18. Lichtman DM, Schneider JR, Swafford AR et al (1981) Ulnar midcarpal instability - clinical and laboratory analysis. J Hand Surg [Am] 6:515-523
19. Lichtman DM, Bruckner JD, Culp RW et al (1993) Palmar midcarpal instability: results of surgical reconstruction. J Hand Surg [Am] 18:307-315

20. Linscheid RL, Dobyns JH (1989) Carpal instability. Curr Orthop 3:106-114

21. Linscheid RL, Dobyns JH, Beabout JW et al (1972) Traumatic instability of the wrist. Diagnosis, classification, and pathomechanics. J Bone Joint Surg Am 54:1612-1632

22. Mayfield JK (1980) Mechanism of carpal injuries. Clin Orthop 149:45-54

23. Navarro A (1935) Anales del Instituto de Clinica Quirurgica y cirugia experimental. Imprenta Artistica de Dornaleche Hnos, Montevideo

24. Pin PG, Nowak M, Logan SE et al. (1990) Coincident rupture of the scapholunate and lunotriquetral ligaments without perilunate dislocation: pathomechanics and management. J Hand Surg [Am] 15(1):110-119

25. Ruby LK (1996) Carpal instability. Instr Course Lect 45:3-13

26. Stanley JK, Trail IA (1994) Carpal instability. J Bone Joint Surg Br 76:691-700

27. Tang JB (1992) Carpal instability associated with fracture of the distal radius. Incidence, influencing factors and pathomechanics. Chin Med J (Engl) 105:758-765

28. Watson HK, Weinzweig J, Zeppieri J (1997) The natural progression of scaphoid instability. Hand Clin 13:39-49

29. Weber ER (1980) Biomechanical implications of scaphoid waist fractures. Clin Orthop 149:83-89

30. Weber ER (1984) Concepts governing the rotational shift of the intercalated segment of the carpus. Orthop Clin North Am 15:193-207

31. Weiss AP (1998) Scapholunate ligament reconstruction using a bone-retinaculum-bone autograft. J Hand Surg [Am] 23(2):205-215

32. Wilhelm A (1965) [Denervation of the wrist.] Hefte Unfallheilkd 81:109-114 